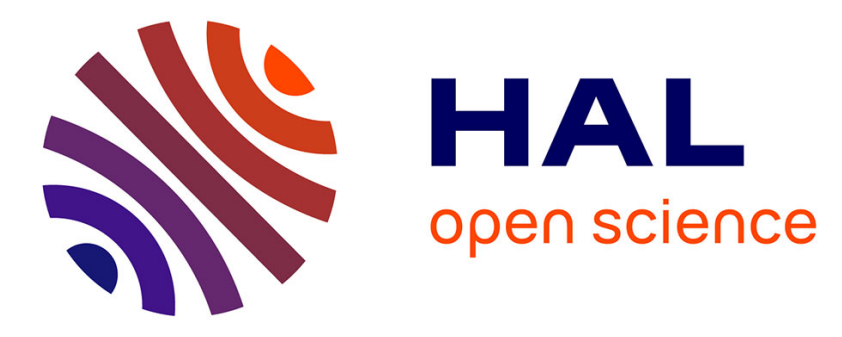

\title{
Ultrafast Optical Mapping of Nonlinear Plasmon Dynamics in $\mathrm{Cu} 2-\mathrm{x}$ Se Nanoparticles
}

Giuseppe Della Valle, Francesco Scotognella, Ajay Ram Srimath Kandada, Margherita Zavelani-Rossi, Dirk Dorfs, Karol Miszta, Matteo Conforti, Stefano Longhi, Liberato Manna, Guglielmo Lanzani, et al.

\section{To cite this version:}

Giuseppe Della Valle, Francesco Scotognella, Ajay Ram Srimath Kandada, Margherita Zavelani-Rossi, Dirk Dorfs, et al.. Ultrafast Optical Mapping of Nonlinear Plasmon Dynamics in Cu 2- x Se Nanoparticles. Journal of Physical Chemistry Letters, 2013, 4 (19), pp.3337-3344. 10.1021/jz401862v . hal02394343

\section{HAL Id: hal-02394343 \\ https://hal.science/hal-02394343}

Submitted on 4 Dec 2019

HAL is a multi-disciplinary open access archive for the deposit and dissemination of scientific research documents, whether they are published or not. The documents may come from teaching and research institutions in France or abroad, or from public or private research centers.
L'archive ouverte pluridisciplinaire HAL, est destinée au dépôt et à la diffusion de documents scientifiques de niveau recherche, publiés ou non, émanant des établissements d'enseignement et de recherche français ou étrangers, des laboratoires publics ou privés. 


\title{
Ultrafast optical mapping of nonlinear plasmon
}

\section{dynamics in $\mathrm{Cu}_{2-\mathrm{x}}$ Se nanoparticles}

\author{
Giuseppe Della Valle, ${ }^{*, \dagger}$ Francesco Scotognella, ${ }^{\dagger}$ Ajay Ram Srimath Kandala, ${ }^{\dagger}$ \\ Margherita Zavelani, ${ }^{\dagger}$ Dirk Dorfs, ${ }^{\ddagger}$ Karol Miszta, ${ }^{\ddagger}$ Matteo Conforti, ${ }^{\text {II }}$ Stefano \\ Longhi, ${ }^{\dagger}$ Liberato Manna, ${ }^{\ddagger}$ Guglielmo Lanzani, ${ }^{\dagger}$ and Francesco Tassone ${ }^{\S}$
}

Politecnico di Milano, Dipartimento di Fisica and Istituto di Fotonica e Nanotecnologie CNR, Piazza Leonardo da Vinci 32, 20133 Milano, Italy, Istituto Italiano di Tecnologia, Via Morego 30, 16163 Genova, Italy, Universitá di Brescia, Dipartimento di Ingegneria dell'Informazione and CNISM, Via Branze 38, 25123 Brescia, Italy, and CNST of IIT@ POLIMI, Via Pascoli 70/3, 20133 Milano, Italy

E-mail: giuseppe.dellavalle@polimi.it

\begin{abstract}
We report on the experimental investigation and theoretical modelling of the ultra-fast nonlinear optical response exhibited by $\mathrm{Cu}_{2-\mathbf{x}} \mathrm{Se}$ nanoparticles in a broad range of wavelengths from the red to the near-infrared under excitation with fs laser beams. The ultra-fast dynamics of the localized plasmon resonance of the system is mapped in a broad spectral region with $\sim 100$ fs resolution time by pump-probe differential transmission measurements. Deviations from theoretical predictions based on standard two-temperature model (TTM) are revealed in

\footnotetext{
*To whom correspondence should be addressed

†Politecnico di Milano

Istituto Italiano di Tecnologia of Genova

IUniversitá di Brescia

§CNST of IIT@POLIMI
} 
the sub-ps time-scale where the nonlinear phenomenon is more pronounced. The key-role played by non-thermalized carriers is investigated in detail on the basis of an extended TTM. 


\section{Introduction}

Plasmon-polaritons in metals ${ }^{1}$ have opened unprecedented avenues for nanoscale manipulation of light, with many exciting applications from sensing to waveguiding. ${ }^{2}$ Most of research have dealed with the linear optical properties of plasmonic systems, but during the very last years the nonlinear response exhibited by plasmonic nanostructures have received an increasing attention (see the fresh review by Kauranen and Zayats ${ }^{3}$ and references therein). Exploitation of the giant optical nonlinearity exhibited by metallic media combined with the unique linear optical features (localization, field-enhancement, etc.) of the plasmon-polariton resonances can disclose a novel frontier for plasmonics. Dynamic plasmonic devices have been proposed and experimentally demonstrated, showing the feasibility of ultra-fast all-optical switching ${ }^{4}$ as well as novel approaches for nanosensing based on plasmonic nonlinearity. ${ }^{5}$ Strong efforts are now being carried on in different directions to improve the efficiency of the nonlinear process in plasmonic structures. One research line is aimed to optimizing the linear part of the phenomenon, that is plasmonic resonances, by seeking for the best nanostructure geometry capable of full exploitation of the nonlinearity in noble metals by increasing the optical absorption or by narrowing the plasmonic resonances (e.g. in arrays of gold nanopillars ${ }^{6}$ ). Another important issue is to provide an accurate modelling of the plasmon dynamics at the level of a single noble-metal nanostructure, ${ }^{7}$ and to reframe metal nonlinearity within a formal non-instantaneous incoherent susceptibility (a dispersed complex $\left.\chi^{(3)}\right)^{8}$ to be exploited for the analysis and engineering of nonlinear propagation of plasmonic pulses. ${ }^{9} \mathrm{~A}$ different approach has been recently started by working on the intimate physics underlying material nonlinearity, thus opening a material route to nonlinear plasmonics. Aside with gold and silver, a novel class of artificial plasmonic media based on heavily-doped semiconductors has been recently reported. ${ }^{10-13}$ These materials exhibit a metallic behaviour with a free-carrier density that can be chemically controlled by acting on fabrication parameters, causing the plasma frequency and thus the plasmonic resonance to be tuned in a broad wavelength range. Most importantly, since the nonlinearity exhibited by metallic systems is governed by the dynamics of the free carriers optically excited, these materials offer the unique capability to enhance the nonlinear response 
of the plasmonic resonance by controlling the free carriers density. $\mathrm{Cu}_{2-\mathbf{x}} \mathrm{Se}$ is one of these novel plasmonic materials, ${ }^{10}$ where the free carriers are holes in the valence band generated by copper vacancies (self-doping mechanism) responsible to the sub-stoichiometry of the compound, with $x$ ranging between 0.1 and 0.3 . In a recent paper we have demonstrated that intense laser beams can strongly perturb the energy distribution of the holes inducing a modulation of the absorption crosssection of $\mathrm{Cu}_{2-\mathbf{x}} \mathrm{Se}$ nanoparticles that is ten times larger than that obtained in gold nanoparticles under the same optical fluence. ${ }^{14}$ We showed that this magnification of the optical nonlinearity is due to the lower density of the free carriers as compared to gold, and a phenomenological model mimicking the simple two-temperature model commonly exploited for gold nanostructures turned out to provide a good explanation of the experimental results. The material route to nonlinear plasmonics is currently at a pioneering level and, similarly to what happened for noble-metals, a better understanding of the intimate nature of the material nonlinearity in concomitance with the plasmonic resonance is mostly envisaged for future developments in the field, and full exploitation of these novel materials as nonlinear plasmonic media.

In the present paper we report an a detailed experimental investigation of the ultra-fast nonlinear response exhibited by $\mathrm{Cu}_{2-x} \mathrm{Se}$ nanoparticles in the red and near-infrared under excitation with fs laser beams. The onset of the plasmon dynamics is spectrally resolved by ultra-fast pumpprobe spectroscopy with broad-band probe pulses. The whole temporal dynamics of carrier-lattice interaction is temporally resolved with 100 fs resolution, allowing for an optical mapping of the plasmonic nonlinear response of $\mathrm{Cu}_{2-x} \mathrm{Se}$ nanoparticles with unprecedented temporal and spectral resolution. Also, we have theoretically developed the preliminary proposal that the dynamical features of localized plasmon resonances in $\mathrm{Cu}_{2-x} \mathrm{Se}$ can be interpreted according to a theoretical approach mimicking the one exploited for noble-metal plasmonic systems, and in particular we considered here an extended version of the two-temperature model (ETTM) that is reminiscent of the one originally proposed for gold by Sun and coworkers. ${ }^{15}$ The combination of broad-band pump-probe measurements and the ETTM allowed us to access the temporal dynamics of nonthermalized carries taking place on the sub-ps time scale, that has been disregarded in all previous 
studies on non-metallic plasmonic nanoparticles. The contribution of these out-of equilibrium carriers to the optical response of the system is accurately modeled in the whole spectrum of the localized plasmon resonance by semiclassical theory of optical transitions in the solid-state, and turned out to dominate the peak of the nonlinear optical response exhibited by $\mathrm{Cu}_{2-x}$ Se nanoparticles.

\section{Experiments}

It has been demonstrated that in $\mathrm{Cu}_{2-x} \mathrm{Se}$ nanocrystals the value of $x$ can be accurately controlled by proper oxidation during the chemical synthesis, allowing tunability of the plasma frequency $\omega_{P}$ of the material, resulting in a tunable Drude dielectric constant and subsequent tunable localized plasmon resonance. ${ }^{10}$ In the present study we selected a sample with a high value of $x$, that is $x \simeq 0.25$, resulting in a plasmonic resonance peaked at $830 \mathrm{~nm}$ for $\mathrm{Cu}_{1.75}$ Se nanocrystals in toluene dispersion (dielectric constant of the environmental medium $\varepsilon_{m}=2.24$ ). The size of $\mathrm{Cu}_{1.75} \mathrm{Se}$ nanocrystals was estimated from transmission electron microscopy and turned out to be of $\sim 6.5 \mathrm{~nm}$ radius.

To investigate the transient nonlinear response of the system, we performed pump-probe experiments with fs-laser pulses. The pump-probe setup [Fig. 1(a)] is based on a commercial Ti:sapphire amplified laser system delivering $100 \mathrm{fs}$ pulses at $1 \mathrm{kHz}$ repetition rate at a central wavelength of $800 \mathrm{~nm}$. A fraction of the beam was used to pump a non-collinear parametric amplifier (NOPA) to generate pulses in the near-infrared. We tuned the NOPA to obtain pump pulses at $\sim 960 \mathrm{~nm}$ with a bandwidth of about $20 \mathrm{~nm}$ [Fig. 1(b)]. The probe pulses were produced by focusing the fundamental beam into a $3 \mathrm{~mm}$ thick sapphire plate in order to generate a stable white light supercontinuum. To generate white light in the visible range [Fig. 1(c)], a short pass filter with cut-off wavelength at $780 \mathrm{~nm}$ was used, while to generate white light in the near infrared range [Fig. 1(d)], a long pass filter with cut-on wavelength at $840 \mathrm{~nm}$ was used to filter out the residual fundamental and the visible components of the probe pulses. The pump and probe beams were focused onto the 
sample with a spot size of $200 \mu \mathrm{m}$. The pump-probe setup employed a computer-controlled optical
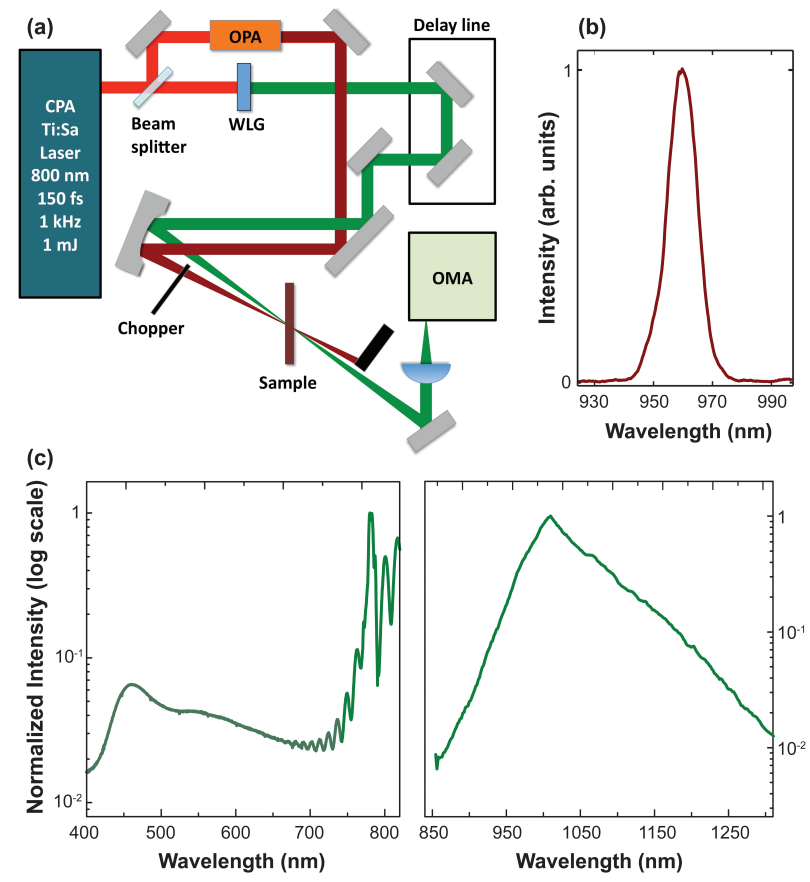

Figure 1: (a) Schematic of the experimental setup. OPA: Optical Parametric Amplifier. WLG: Wight Light Generator. OMA: Optical Multichannel Analyzer. (b) Pump pulse spectrum. (c) Probe pulse spectra.

multichannel analyzer and the measured signal is a map of the chirp-free differential transmission $\Delta T / T=\left(T_{o n}-T_{o f f}\right) / T_{o f f}$ as a function of the pump-probe time delay for a broad spectrum of probe wavelengths; $T_{o n}$ and $T_{o f f}$ are the probe spectra transmitted by the excited and unperturbed samples, respectively.

Differential transmission measurements were attained at $150 \mu \mathrm{J} / \mathrm{cm}^{2}$ incident pump fluence. Since we pumped $130 \mathrm{~nm}$ detuned from the peak of the plasmon resonance, the measured pump absorption turned out to be as low as $8 \%$, which is markedly lower as compared to the previous study on $\mathrm{Cu}_{1.85}$ Se nanocrystals ${ }^{14}$ where the pump wavelength was at the peak of the plasmonic resonance. Experimental $\Delta T / T$ map is reported in Fig. 2(a). Note that the transient spectral response is composed of three different lobes, a stronger and broader positive lobe in the central region of the spectrum, and two weaker negative side-lobes (below $650 \mathrm{~nm}$ and above $900 \mathrm{~nm}$ respectively). A maximum value of about $3.5 \%$ is observed at around $770 \mathrm{~nm}$, whereas minimum values of $-1 \%$ and $-0.5 \%$ are attained in the negative lobes at around $560 \mathrm{~nm}$ and $1010 \mathrm{~nm}$ respectively. These 

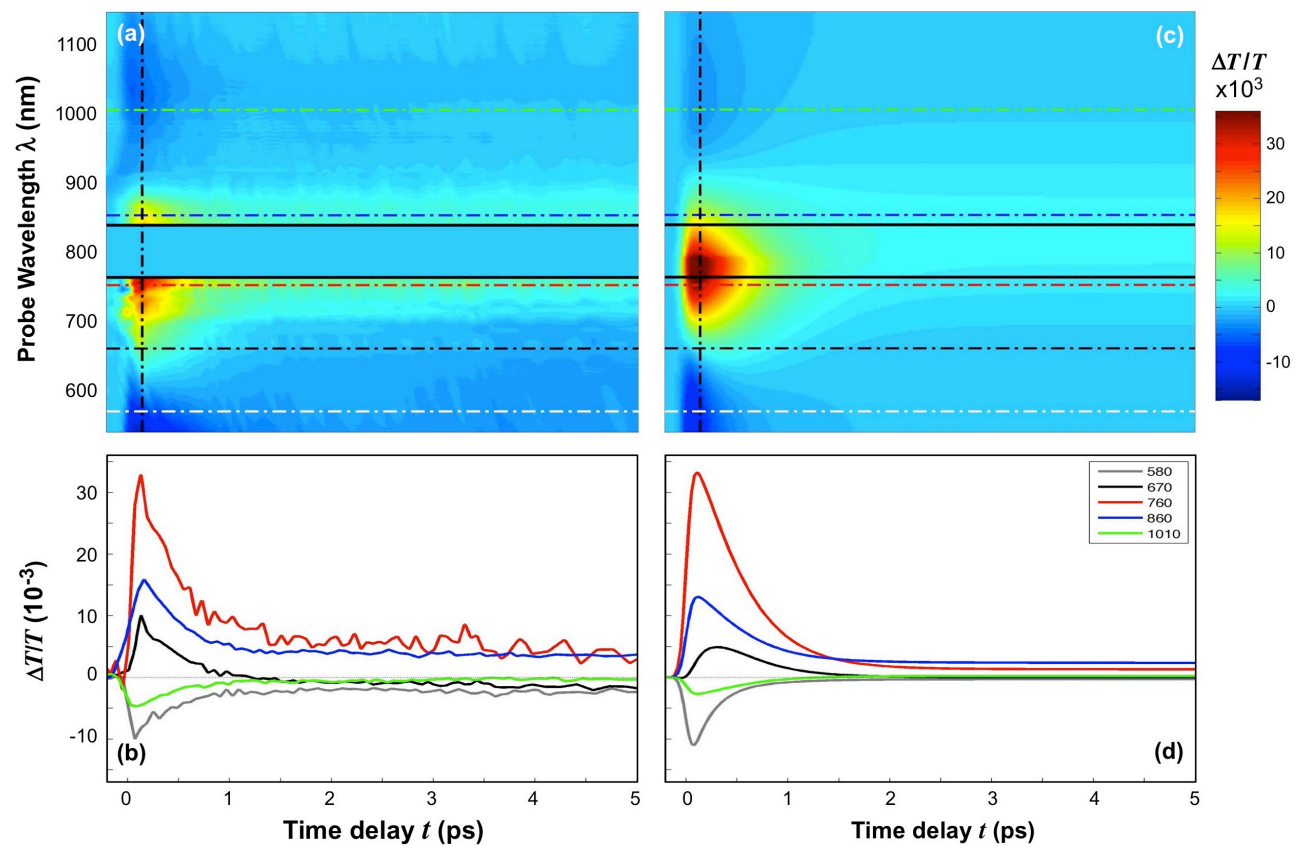

Figure 2: (a) Experimental Differential Transmission map of $\mathrm{Cu}_{1.75} \mathrm{Se}$ nanocrystals in toluene. (b) Temporal cross-sections of the experimental map in (a) at different probe-wavelengths. (c) Numerically computed Differential Transmission map according to the ETTM. (d) Temporal crosssections of the theoretical map in (c) at different probe-wavelengths.

maxima and minima are attained at approximately $150 \mathrm{fs}$ time delay, after which a monotonic and fast decrease of the signal is observed within 2 picoseconds [see also Fig. 2(b)] in agreement with previous results of single wavelength probing. ${ }^{14}$ The following dynamics is much slower, with an almost exponential decay within few hundred ps (not shown here) as already reported in. ${ }^{14}$

\section{Theoretical analysis and numerical modelling}

For nanoparticles with radius $R<<2 \pi c / \omega$ (with $\omega$ the optical frequency), optical extinction is dominated by absorption, with absorption cross-sections $\sigma_{A}$ given by the quasi-static formula ${ }^{16}$

$$
\sigma_{A}=4 \pi k R^{3} \operatorname{Im}\left\{\frac{\varepsilon(\omega)-\varepsilon_{m}}{\varepsilon(\omega)+2 \varepsilon_{m}}\right\}
$$

where $\varepsilon(\omega)=\varepsilon_{\infty}-\omega_{P}^{2} /\left[\omega\left(\omega+i \Gamma_{0}\right)\right]$ is the $\mathrm{Cu}_{1.75}$ Se dielectric function, with $\Gamma_{0}$ the Drude damping at the room temperature $T_{0}$ and $\varepsilon_{\infty}$ a constant accounting for interband transitions at higher 
optical frequencies. Similarly to previous studies, the values of $\omega_{P}, \Gamma_{0}$, and $\varepsilon_{\infty}$, have been estimated from continuous-wave extinction measurements of the sample, ${ }^{14}$ and turned out to be $\omega_{P}=8.58 \times 10^{15} \mathrm{rad} / \mathrm{s}, \Gamma_{0}=10.1 \times 10^{14} \mathrm{rad} / \mathrm{s}$, and $\varepsilon_{\infty}=10$. The transmittance of the system can thus be computed as $T=\exp \left[-\sigma_{A} N_{p} L\right]$, with $N_{p}=12.9 \times 10^{13} \mathrm{~cm}^{-3}$ being the estimated particle concentration and $L=0.5 \mathrm{~mm}$ the thickness of the sample which is hold in a quartz cuvette. Excitation with intense (pump) laser pulses gives rise to a transient modification of the absorption cross-section of the nanocrystals $\Delta \sigma_{A}(t)$, resulting in a transient modulation of the transmittance of the sample given by

$$
\Delta T(t)=T\left\{\exp \left[-\Delta \sigma_{A}(t) N_{p} L\right]-1\right\}
$$

To provide a consistent spectral modelling of the measured differential transmission retrieved by pump-probe experiments, a model for the transient variation of absorption cross-section resulting from the variation of material dielectric function caused by pump absorption ought to be provided. In noble metal nanoparticles the variation of metal dielectric function is ascribed to the dynamics of energy exchange between free carriers (electrons) and lattice, that is detailed by the so-called twotemperature model (TTM). To account for non-thermalized carriers we adopted an extended version of the TTM (ETTM) that has been proposed and successfully exploited for thin-gold films ${ }^{15}$ and nanoparticles (see e.g. pioneering pump-probe experiments on gold nanoshells ${ }^{17}$ ). The ETTM reads as follows:

$$
\begin{aligned}
\gamma T_{C} \frac{d T_{C}}{d t} & =-G\left(T_{C}-T_{L}\right)+a N \\
C_{L} \frac{d T_{L}}{d t} & =G\left(T_{C}-T_{L}\right)-G_{L}\left(T_{L}-T_{0}\right)+b N \\
\frac{d N}{d t} & =-(a+b) N+P_{a}(t)
\end{aligned}
$$

where $T_{C}$ is the temperature of thermalized free carriers (i.e. holes in $\mathrm{Cu}_{1.75} \mathrm{Se}$ ), $T_{L}$ is the lattice temperature, $N$ is the total energy per unit volume of non-thermalized carriers, $\gamma T_{C}$ and $C_{L}$ are the free carrier and lattice heat capacities, respectively (being $\gamma$ the so-called carrier heat capacity constant), $G$ is the carrier-phonon coupling constant, $G_{L}$ is the phonon-phonon coupling constant, 
$a$ and $b$ are the carrier and lattice heating rates respectively, and $P_{a}(t)$ is the pump pulse power absorbed in the unit volume of the metal. Parameters for the ETTM were taken from previous estimation ${ }^{14}$ on $\mathrm{Cu}_{1.85} \mathrm{Se}$ as $C_{L}=2.72 \cdot 10^{6} \mathrm{~J} \mathrm{~m}^{-3} \mathrm{~K}^{-1}, G=1.2 \cdot 10^{16} \mathrm{~W} \mathrm{~m}^{-3} \mathrm{~K}^{-1}$, and $G_{L}=2.0 \cdot 10^{16}$ $\mathrm{W} \mathrm{m}{ }^{-3} \mathrm{~K}^{-1}$. The heat capacity constant of the carriers was estimated as $\gamma=r \gamma_{A u}=5.1 \mathrm{~J} \mathrm{~m}^{-3} \mathrm{~K}^{-2}$, with $\gamma_{A u}=63 \mathrm{~J} \mathrm{~m}^{-3} \mathrm{~K}^{-2}$ the heat capacity constant of the free electrons in gold and $r$ the ratio between $\mathrm{Cu}_{1.75} \mathrm{Se}$ carrier density $N_{C}$ and gold carrier density $\left(5.9 \times 10^{22} \mathrm{~cm}^{-3}\right)$. We estimated $N_{C}=\omega_{P} \varepsilon_{0} m_{h} / e^{2}=4.78 \times 10^{21} \mathrm{~cm}^{-3}$, taking the hole effective mass $m_{h}=0.20 m_{0}$ (being $m_{0}$ the free electron mass) as reported in the pioneering paper on $\mathrm{Cu}_{2-x} \mathrm{Se}$ by Gorbachev and Putilin. ${ }^{20}$ Within the present study, $a$ is considered as a fitting parameter of the model, and we assumed $b \simeq 0$ similarly to what reported in noble metals where $b<<a$.

It is worth saying that the ETTM is the simplest model that accounts for the carrier-carrier interaction mechanism (that is rigorously described by Boltzmann equations), by grasping the essentials of its dynamical features in terms of an effective averaged parameter $(N)$ for the nonthermalized part of the system. The ETTM offers the advantage to keep the modeling at the level of three coupled-equations (and sometimes it is referred to as a three-temperature model, though this nomenclature is misleading since the new variable $N$ is not a temperature) governed by very few phenomenological parameters.

The system of Eqs. (4)-(5) allows one to consistently calculate the temporal evolution of $T_{C}$ and $N$ and thus the corresponding contribution to the variation of the thermalized $\Delta f_{T}$ and nonthermalized $\Delta f_{N T}$ free-carriers distributions, respectively given by: ${ }^{15}$

$$
\begin{aligned}
\Delta f_{T}(E, t) & =f_{0}\left[E, T_{C}(t)\right]-f_{0}\left(E, T_{0}\right) \\
\Delta f_{N T}(E, t) & =1 / A \times \delta_{N T}(E) N(t),
\end{aligned}
$$

being $f_{0}$ the Fermi-Dirac function, $E$ the holes energy, and

$$
\delta_{N T}(E)=f_{0}\left(E-E_{F}-h v_{P}\right)\left[1-f_{0}\left(E-E_{F}\right)\right]-f_{0}\left(E-E_{F}\right)\left[1-f_{0}\left(E-E_{F}+h v_{P}\right)\right]
$$


In above Eqs. (7)-(8), $E_{F}$ is the (holes) Fermi Energy, $h v_{P}$ is the pump photon energy, and A is a normalization factor determined according to energy conservation for the intra-band pump absorption, that is

$$
A=\int_{E_{1}}^{E_{2}} \delta_{N T}(E) D O S(E) E d E
$$

with $\operatorname{DOS}(E)$ the energy density of states in the valence band under isotropic parabolic band approximation. Integration extrema are taken to be $E_{1}$ the energy at the edge of the valence band and $E_{2}$ the energy of hole states with $k$-vector at half the maximum $k$ in the first Brillouin zone (as an upper limit for the application of parabolic band approximation).

Note that Eq. (8) provides for non-thermalized holes generated by intra-band pump absorption a double steplike distribution (see, e.g., Ref. ${ }^{15}$ ) extending from $-h v_{P}$ to $+h v_{P}$ around Fermi energy. The total variation of the electron energy distribution due to thermal and non-thermal holes results in a reduction (increase) of the occupation probability of the hole states below (above) the Fermi energy. Since according to Al-Mamun et al., ${ }^{18}$ the $\mathrm{Cu}_{2-x} \mathrm{Se}$ system is a direct band-gap material, the pump-induced variation of occupation probability of energy states close to $E_{F}$ is expected to result in a modulation of the inter-band optical transition, with increased (decreased) absorption for transitions involving final hole states below (above) $E_{F}$. In the constant matrix element approximation, the variation of the imaginary part of the inter-band dielectric function at the probe wavelength $\lambda$ due to the pump-induced perturbation of thermal (T) and non-thermal (NT) hole energy distributions can be computed as follows: ${ }^{19}$

$$
\Delta \varepsilon_{T(N T)}^{\prime \prime}(\lambda, t)=\frac{e^{2} \lambda^{2}}{3 m_{0}^{2} c^{2}}|M|^{2} \Delta J_{T(N T)}(\lambda, t)
$$

where $M$ is the electric-dipole matrix element, that we consider as a fitting parameter in our model, and $\Delta J_{T(N T)}$ is the pump-induced variation of the Joint Density of States (JDOS) of the considered transition:

$$
\Delta J_{T(N T)}(\lambda, t)=-\frac{2}{4 \pi^{2}}\left(\frac{2 m_{r}}{\hbar^{2}}\right)^{3 / 2} \sqrt{\frac{h c}{\lambda}-E_{0}} \times \Delta f_{T(N T)}\left(E_{\lambda}, t\right)
$$


In above Eq. (11), $E_{\lambda}=m_{r} / m_{h}\left(h c / \lambda-E_{0}\right)-E_{F}$ according to energy conservation, with $m_{r}=$ $m_{h} m_{e} /\left(m_{h}+m_{e}\right)$ the reduced effective mass of the system, and $m_{e}$ the electron effective mass in the conduction band, here assumed as a fitting parameter of the model. The energy constants are taken from our previous study ${ }^{14}$ as $E_{0}=1.55 \mathrm{eV}$ for the energy gap between valence and conduction bands and $E_{F}=0.6 \mathrm{eV}$ for the Fermi energy measured from the top of valence band [cf. Fig. 3(a) in Ref. ${ }^{14}$ ].

With $\Delta \varepsilon_{T(N T)}^{\prime \prime}(\lambda, t)$ at hand, the variation of the real part of the inter-band dielectric function $\Delta \varepsilon_{T(N T)}^{\prime}(\lambda, t)$ is determined by Kramers-Krönig analysis.

An additional contribution to the change in the dielectric constant, given by intra-band transitions of free carriers that depend on lattice temperature (i.e. the Drude contribution), is accounted for by the following expression:

$$
\Delta \varepsilon_{D S}(\lambda, t)=\left.\frac{i \omega_{P}^{2} \Delta \Gamma(t)}{\omega\left(\omega+i \Gamma_{0}\right)\left[\omega+i \Gamma_{0}+i \Delta \Gamma(t)\right]}\right|_{\omega=2 \pi c / \lambda}
$$

where $\Delta \Gamma(t)=\beta\left[T_{L}(t)-T_{0}\right]$ is the variation of the Drude damping induced by lattice temperature variation, being $\beta=1.8 \times 10^{12} \mathrm{rad} / \mathrm{s} \mathrm{K}^{-1}$ as estimated in our previous study. ${ }^{14}$ From the timedependent complex dielectric function of $\mathrm{Cu}_{1.75}$ Se provided by Eqs. (10)-(12), we theoretically computed the time-dependent variation $\Delta \sigma_{A}(\lambda, t)$ attained by the absorption cross section according to standard quasi-static formula of Eq. (1). Then, the differential transmission of the sample is retrieved from Eq. (2).

Results of the numerical simulations with best fitting value of the parameters are reported in Fig. 2(c)-(d), and show a good quantitative agreement with the experimental data of Fig. 2(a)-(b) in the wide range of wavelengths spanned to inspect the whole spectrum of the plasmon resonance. Best fitting of parameters retrieved the following estimations: electrons effective mass $m_{e} \simeq 0.12 m_{0}$; electric-dipole matrix element $M \simeq 2 M_{A u}$ being $M_{A u}$ the electric-dipole matrix element for interband optical transitions in gold at the $L$-point of the Brillouin zone; ${ }^{21}$ carrier heating rate $a=2.4 \times 10^{12} \mathrm{~s}^{-1}$. 


\section{Discussion}

The numerical solution of the ETTM system of Eqs. (4)-(5) is reported in Fig. 3(a). The maximum of energy density of non-thermalized holes turned out to be $N_{\max } \simeq 1.1 \mathrm{~J} / \mathrm{cm}^{3}$, and it is achieved at around 80 fs time delay, whereas the maximum increase in the temperature of the thermalized holes is about $150 \mathrm{~K}$ (i.e. lower than the room temperature, meaning that we are in the perturbative regime for pump absorption), and it is achieved at around $250 \mathrm{fs}$. The increase in the lattice temperature is as low as $0.5 \mathrm{~K}$, in light of the much higher heat capacity of the lattice as compared to the holes, and is achieved after about 3 ps. Note that the very initial steps of the dynamics are dominated by non-thermalized carriers. On the contrary the lattice plays no role until the dynamics of non-thermalized holes is almost exhausted. In the intermediate regime comprised between 0.5 ps to $3 \mathrm{ps}$, the dynamics is dominated by thermalized carriers. The sub-ps time scale is where the hole-hole scattering regime takes place and the optical response of the system is determined by the interplay between non-thermalized and thermalized holes with a complex spectral behaviour governed by Eqs. (10)-(12). To elucidate the role played by non-thermalized carriers, we show in Fig. 3(b) and (c) the theoretically computed contributions to $\Delta T / T$ arising from thermalized and non-thermalized holes at two different probe wavelengths: one in the positive lobe of the $\Delta T / T$ map, at $860 \mathrm{~nm}$ [Fig. 3(b)], and one in the lower negative lobe of the $\Delta T / T$ map, at $580 \mathrm{~nm}$ [Fig. 3(c)].

At $860 \mathrm{~nm}$, that is above the peak of the plasmonic resonance, both thermalized and nonthermalized holes provide a positive contribution to $\Delta T / T$, resulting in a large positive peak of the total $\Delta T / T$ [Fig. 3(b)]. A similar dynamics (not detailed here) is attained below the peak of the plasmonic resonance remaining in the positive lobe of the $\Delta T / T$ map (in agreement with our previous study ${ }^{14}$ ), but a very distinct behaviour is observed in the lower negative lobe of the map. As example, at $580 \mathrm{~nm}$ the thermalized carriers again provide a positive signal, but nonthermalized ones give rise to a large negative contribution, resulting in a negative peak of the total $\Delta T / T$ [Fig. 3(c)]. The role of non-thermalized carriers is thus not just quantitative, by providing a correction to the dynamics within the initial few hundred fs, but it's crucial in determining 

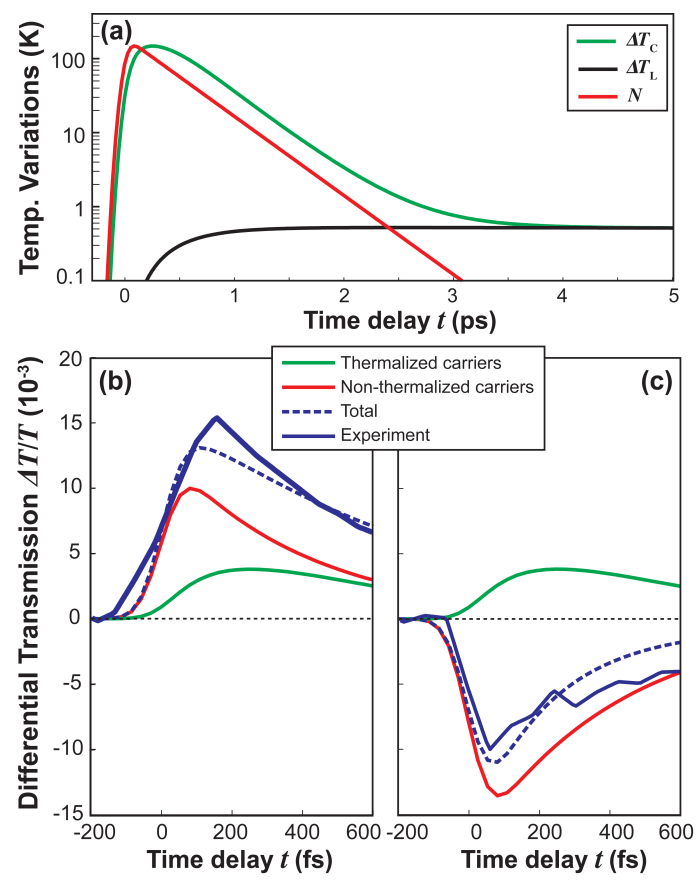

Figure 3: (a) Temporal dynamics of thermalized carrier temperature $T_{C}$ (green line), lattice temperature $T_{L}$ (black line), and non-thermalized electron energy density $N$ normalized to peak value $N_{\max }=1.1 \mathrm{~J} / \mathrm{cm}^{3}$ (red line). Contributions to the differential transmission signal arising from thermalized and non-thermalized carriers at (b) $860 \mathrm{~nm}$ and (c) $580 \mathrm{~nm}$ probe wavelengths. The total theoretical $\Delta T / T$ (blue dotted line) and experimental $\Delta T / T$ (blue solid line) are also reported. 
wheather the pump-induced modulation of probe transmission is positive or negative at a given optical wavelength.

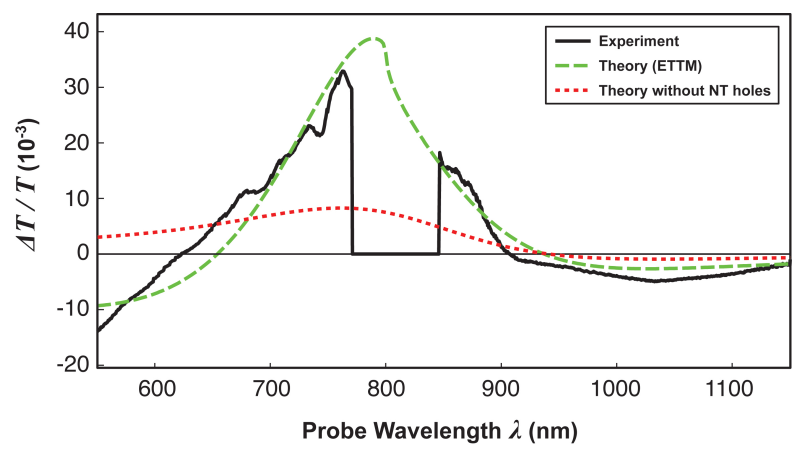

Figure 4: Spectral cross-section of the $\Delta T / T$ experimental map (solid black) and theoretical map (dashed green) at peak time delay $t \simeq 150$ fs. Dotted red line shows the theoretical prediction neglecting the contribution from non-thermalized carriers.

The importance of including non-thermalized carriers for a correct determination of the spectral response of the system is even more evident if one inspects the $\Delta T / T$ spectrum at the time delay of the peak, attended at around $150 \mathrm{fs}$ [see vertical black dot-dashed line in Fig. 2(a) and (c)]. Note that the theoretical $\Delta T / T$ (green dashed line in Fig. 3) well reproduces the experimental data (black solid line in Fig. 3) in the whole range of the plasmonic resonance, with only some deviations in the tails of the spectrum. On the contrary, neglecting the contribution from non-thermalized carriers results in a theoretical prediction (red dotted line in Fig. 3) with dramatic deviations from the experimental results at almost any wavelength.

It is worth saying that the main limitations of the present model arise from the isotropic parabolic band and constant matrix element approximations exploited to compute the modulation of interband optical transition. This prevented to retrieve a quantitative agreement in the red and visible (not shown here). For further developments, a detailed knowledge of $\mathrm{Cu}_{1.75} \mathrm{Se}$ band structure is mandatory. A theoretical investigation of the electronic properties of non-stoichiometric $\mathrm{Cu}_{2} \mathrm{Se}$ would also give access to a more accurate modeling of the sub-ps time-scale dynamics within Boltzmann equations formalism, as reported in several gold nano-systems. ${ }^{15,22,23}$ At the level of the present modelling we just notice that the estimated value of carrier heating rate $a$ turned out to be almost coincident with what reported in gold. ${ }^{8,15}$ Since it has been demonstrated that $a=1 / 2 \tau_{1}$, 
with $\tau_{1}$ being the minimum value of the electron-electron relaxation time within the population of non-thermalized carriers, ${ }^{22}$ we can estimate $\tau_{1} \simeq 200 \mathrm{fs}$.

Also, we noted some quantitative mismatch between experiments and simulations in the long timescale, indicating that the Drude contribution to the dielectric function modulation provided by Eq. (12) is incomplete. A possible extension would comprise the effect of lattice temperature on the plasma frequency caused by lattice deformation, especially in connection to mechanical oscillations on the ns time-scale induced by the abrupt thermal loading, as observed in noble-metal nanoparticles (see e.g. ${ }^{24}$ and references therein).

\section{Conclusions}

We investigated the ultra-fast nonlinear dynamics exhibited by localized-plasmon resonance in $\mathrm{Cu}_{2-x} \mathrm{Se}$ nanoparticles. Broad-band pump-probe experiments with 100 fs resolution time provided an accurate tracking of the phenomenon in two-dimensional maps spannig the whole plasmonic spectrum of the resonance on the time scale of electron-lattice interaction dynamics. An accurate modelling of the experimental results based on an extended two temperature model and semiclassical computation of the optical transitions was carried out within quasi-static approximation, in close analogy with the dynamical models employed for noble-metal nanostructures. A comparison between experiments and theoretical results revealed the crucial role played by nonthermalized carriers during the very first initial steps of the dynamics, with an overshoot to the differential transmission in a broad wavelength range that the standard two-temperature model dramatically fails to retrieve. Our results provide a detailed analysis and theoretical interpretation of the ultra-fast dynamics in an artificial plasmonic medium, and demonstrate that the theoretical models developed for noble-metal nanostructures can be fully exploited for $\mathrm{Cu}_{2-x}$ Se plasmonic nanoparticles, paving the way for further developments of this new material with potential applications to all-optical ultra-fast switching and nonlinear nanosensing. ${ }^{3}$ 


\section{Methods}

\section{Materials}

\section{Numerical simulations}

\section{Acknowledgement}

G. Della Valle and S. Longhi acknowledge financial support from the Fondazione Cariplo through the project New Frontiers in Plasmonic Nanosensing (Grant No. 2011-0338).

\section{References}

1. Raether, H. Surface Plasmons, 1st ed.; Springer-Verlag: Berlin, 1986.

2. Lal, S.; Link, S.; Halas, N. J. Nano-optics from sensing to waveguiding. Nat. Photonics 2007, $1,641-648$.

3. Kauranen, M.; Zayats, A. V. Nonlinear plasmonics. Nat. Photonics 2012, 6, 737-748.

4. MacDonald, K. F.; Samson, Z. L.; Stockman, M. I.; Zheludev, N. I. Ultrafast Active Plasmonics. Nat. Photonics 2009, 3, 55-58.

5. Stockman, M. I. Ultrafast nanoplasmonics under coherent control. New J. Phys. 2008, 10, 025031-1-025031-20.

6. Wurtz, G. A.; Pollard, R.; Hendren, W.; Wiederrecht, G. P.; Gosztola, D. J.; Podolskiy, V. A.; Zayats, A. V. Designed ultrafast optical nonlinearity in a plasmonic nanorod metamaterial enhanced by nonlocality. Nat. Nanotechnol. 2011, 6, 107-111.

7. Baida, H.; Mongin, D.; Christofilos, D.; Bachelier, G.; Crut, A.; Maioli, P.; Fatti, N. D.; ; Vallee, F. Ultrafast Nonlinear Optical Response of a Single Gold Nanorod near Its Surface Plasmon Resonance. Phys. Rev. Lett. 2012, 107, 057402-1-057402-5. 
8. Conforti, M.; Valle, G. D. Derivation of third-order nonlinear susceptibility of thin metal films as a delayed optical response. Phys. Rev. B. 2012, 85, 245423-1-245423-4.

9. Marini, A.; Lee, H.; Conforti, M.; Valle, G. D.; Tran, T. X.; Chang, W.; Schmidt, M. A.; Longhi, S.; Russell, P. S. J.; Biancalana, F. Thermo-modulational interband susceptibility and ultrafast temporal dynamics in nonlinear gold-based plasmonic devices. New. J. Phys. 2013, in press.

10. Dorfs, D.; Hartling, T.; Miszta, K.; Bigall, N. C.; Kim, M. R.; Genovese, A.; Falqui, A.; Povia, M.; Manna, L. Reversible Tunability of the Near-Infrared Valence Band Plasmon Resonance in $\mathrm{Cu}_{2-x}$ Se Nanocrystals. J. Am. Chem. Soc. 2011, 133, 11175-11180.

11. Luther, J. M.; Jain, P. K.; Ewers, T.; Alivisatos, A. P. Localized surface plasmon resonances arising from free carriers in doped quantum dots. Nat. Mater. 2011, 10, 361-366.

12. Garcia, G.; Buonsanti, R.; Runnerstrom, E. L.; Mendelsberg, R. J.; Llordes, A.; Anders, A.; Richardson, T. J.; Milliron, D. J. Dynamically Modulating the Surface Plasmon Resonance of Doped Semiconductor Nanocrystals. Nano Lett. 2011, 11, 4415-4420.

13. Manthiram, K.; Alivisatos, A. P. Tunable Localized Surface Plasmon Resonances in Tungsten Oxide Nanocrystals. J. Am. Chem. Soc. 2012, 134, 3995-3998.

14. Scotognella, F.; Valle, G. D.; Kandada, A. R. S.; Dorfs, D.; Zavelani-Rossi, M.; Conforti, M.; Miszta, K.; Comin, A.; Korobchevskaya, K.; Lanzani, G. et al. Plasmon Dynamics in Colloidal $\mathrm{Cu}_{2-x}$ Se Nanocrystals. Nano Lett. 2011, 11, 4711-4717.

15. Sun, C.-K.; Vallee, F.; Acioli, L. H.; Ippen, E. P.; Fujimoto, J. G. Femtosecond-tunable measurement of electron thermalization in gold. Phys. Rev. B 1994, 50, 337-348.

16. Bohren, C. F.; Huffman, D. R. Absorption and Scattering of Light by Small Particles, 1st ed.; John Wiley and Sons: New York, 1983. 
17. Averitt, R. D.; Westcott, S. L.; Halas, N. J. Ultrafast electron dynamics in gold nanoshells. Phys. Rev. B 1998, 58, R10 203-R10 2006.

18. Mamun, A. B. A.; Islam, M. O.; Bhuiyan, A. H. Structural, electrical and optical properties of copper selenide thin films deposited by chemical bath deposition technique. J. Mater. Sci. Mater. Electr. 2005, 16, 263-268.

19. Rosei, R. Temperature modulation of the optical transitions involving the Fermi surface in Ag: Theory. Phys. Rev. B 1974, 10, 474-483.

20. Gorbachev, V. V.; Putilin, I. M. Some Parameters of Band Structure in Copper Selenide and Telluride. Phys. Stat. Sol. (a) 1973, 16, 553-559.

21. Guerrisi, M.; Rosei, R.; Winsemius, P. Splitting of the interband absorption edge in Au. Phys. Rev. B 1975, 12, 557-563.

22. Valle, G. D.; Conforti, M.; Longhi, S.; Cerullo, G.; Brida, D. Real-time optical mapping of the dynamics of nonthermal electrons in thin gold films. Phys. Rev. B 2012, 86, 155139.

23. Voisin, C.; Christofilos, D.; Loukakos, P. A.; Fatti, N. D.; F. Vallee, M. G. E. C. M. P., J. Lerme; Broyer, M. Ultrafast electron-electron scattering and energy exchanges in noble-metal nanoparticles. Phys. Rev. B 2004, 69, 195416.

24. Hartland, G. V. Optical Studies of Dynamics in Noble Metal Nanostructures. Chem. Rev. 2011, $111,3858-3887$. 